\title{
LA ORGANIZACIÓN Y SU ADAPTACIÓN A LAS TECNOLOGÍAS DE LA INFORMACIÓN Y LA COMUNICACIÓN EN PROCESOS DE GESTIÓN DEL CONOCIMIENTO*
}

\author{
Jenny Martínez Crespo** \\ Lillyana María Giraldo Marín*** \\ Recibido: noviembre 23 de 2010 - Aceptado: noviembre 13 de 2012
}

\section{RESUMEN}

El avance de las tecnologías de la información y la comunicación, y su incidencia en el mundo organizacional son innegables. Las organizaciones se han adaptado a las lógicas de interacción, conectividad y acción que estas tecnologías ofrecen. La empresa normal, de administración tradicional, cede terreno a organizaciones con estructuras flexibles, estrategias construidas sobre intangibles que generan valor y culturas que permitan la interacción del colectivo para la gestión del conocimiento. No obstante, la observación y el análisis de esta relación en algunas grandes empresas de la ciudad de Medellín permite determinar que los avances en las tecnologías de la información y la comunicación, como soporte a procesos de gestión del conocimiento, se implementan en estructuras rígidas y piramidales, por fuera de las estrategias y políticas de la organización, y bajo esquemas culturales construidos desde la estandarización, la medición y el control. Surge así, la reflexión en torno a la dificultad implícita de asimilar a la gestión administrativa (estrategia, estructura y cultura), estas tecnologías y proyectos de gestión del conocimiento en las organizaciones. En este sentido, este análisis reflexivo surge de la evaluación del impacto de la implementación de proyectos de gestión del conocimiento en algunas empresas en Antioquia, proyectos soportados en tecnologías de la información y la comunicación.

\section{PALABRAS CLAVE}

Gestión organizacional, gestión del conocimiento, tecnologías de la información y la comunicación.

\section{CLASIFICACIÓN JEL}

M10, M14, M15

\section{CONTENIDO}

Introducción; 1. La empresa normal y la administración tradicional; 2. Tecnologías de la información y la comunicación: herramientas de apoyo para la gestión del conocimiento. 3. Adaptación de la empresa normal a las tecnologías de la información y la comunicación, para apoyar procesos de gestión del conocimiento; 4. Gestión administrativa. Permanente proceso de adaptación; 5. A manera de síntesis (para la discusión); 6. Conclusiones, Bibliografía.

- Este artículo de reflexión se soporta en observaciones realizadas en la investigación titulada "Impacto de la implementación de proyectos de gestión del conocimiento en la creación de valor en algunas grandes empresas en la ciudad de Medellín", financiado por la Vicerrectoría de Investigaciones, Universidad de Medellín. Ejecutado en el periodo enero 2011 -diciembre 2012. Inscrito en el Grupo de Investigación Cultura y Gestión Empresarial (CYGO), de la Facultad de Ciencias Económicas y Administrativas de la Universidad de Medellín.

"* Administradora de Empresas, Universidad de Nariño, Pasto, Colombia. Magíster en Ciencias de la Organización, Universidad del Valle, Cali, Colombia. Magíster en Administración de Empresas, Universidad del Valle, Cali, Colombia. Estudiante del Doctorado en Administración, Universidad EAFIT, Medellín, Colombia. Docente e Investigadora de la Facultad de Ciencias Económicas y Administrativas de la Universidad de Medellín. Miembro del grupo de investigación CYGO. Cra. 87 No. 30-65 Bloque 12-214, Medellín, Colombia. Tel. (57 4) 3405562 Ext. 5225. Correo electrónico: jenny_mar_cre@yahoo.es ó jmartinez@udem.edu.co.

..* Ingeniera de Sistemas, Universidad de Medellín, Colombia. Especialista en Gerencia de la Información Y Magíster en Educación, Universidad de Medellín, Colombia. Candidata a Doctora en Informática, Universidad Pontificia de Salamanca, España. Correo electrónico: Imgiraldo@udem.edu.co. 


\section{THE ORGANIZATION AN ITS ADAPTATION TO INFORMATION AND COMMUNI- CATION TECHNOLOGIES IN THE KNOWLEDGE MANAGEMENT PROCESS}

\section{ABSTRACT}

The advance of information and communication technologies and their impact in the organizational world is undeniable. Organizations have adapted to the logic of interaction, connectivity and actions that these technologies offer. The typical traditionally managed business has a competitive disadvantage compared with businesses that have a flexible structure, strategies constructed on intangible goods that generate value and cultures that enable collective interaction for knowledge management. However, the observation and analysis of this relationship in some companies in the city of Medellin show that technology advancements in information and technology, as support for the knowledge management process, are implemented in rigid and pyramidal structures which are not part of the main policies and strategies of the business and under already constructed cultural schemes based on standardization, measurement and control. Thus arises the reflection on the underlying difficulty of the administrative management (strategy, structure and culture) in applying these technologies in the knowledge management processes in their organizations. In the sense, this analysis emerges form the impact assessment on the implementation of knowledge management processes in some companies of Antioquia; Projects supported on information and communication technologies.

KEY WORDS

Organizational management, knowledge management, information and comunication technology.

JEL CLASSIFICATION

M10, M14, M15, L29

CONTENT

Introduction; 1. Normal businesses and traditional administration; 2. Information and communication technologies. 3. Adaptation of a normal business to information and communication technologies in order to support knowledge management processes; 4. Administrative management. Permanent adaptation process; 5. Synthesis (for discussion); 6. Conclusions, Bibliography.

\section{A ORGANIZAÇÃO E SUA ADAPTAÇÃO À TECNOLOGÍAS DA INFORMAÇÃO E A COMUNICAÇÃOO EM PROCESSOS DE GESTÃO DO CONHECIMENTO}

\section{RESUMO}

O avanço das tecnologias da informação e a comunicação, e sua incidência no mundo organizacional é inegável. As organizações tem-se adaptado à lógicas de interação, conectividade e ação que estas tecnologias oferecem. A empresa normal, de administração tradicional, perde terreio para organizações com estruturas flexíveis, estratégias construídas sobre intangíveis que geram valor e culturas que permitam a interação do coletivo para a gestão do conhecimento. No entanto, a observação e a análises desta relação em algumas grandes empresas da cidade de Medellín, permite determinar que os avanços nas tecnologias da informação e a comunicação, como suporte a processos de gestão do conhecimento, aplicam-se em estruturas rígidas e piramidais, por fora das estratégias e políticas da organização e, sob esquemas culturais construídos desde a estandardização, a medição e o controle. Surge assim, a reflexão em volta à dificuldade implícita de assimilar, à gestão administrativa (estratégia, estrutura e cultura), estas tecnologias a projetos de gestão do conhecimento nas organizações. Neste sentido, esta análise refletiva surge da avaliação do impacto da aplicação de projetos de gestão do conhecimento em algumas empresas em Antioquia; projetos suportados em tecnologias da informação e a comunicação.

PALAVRAS CHAVE

Gestão organizacional, gestão do conhecimento, tecnologias da informação e a comunicação.

CLASIFICAÇÃO JEL

M10, M14, M15, L29

\section{CONTEUDO}

Introdução; 1. A empresa normal e a administração tradicional; 2. Tecnologias da informação e a comunicação: ferramentas de apoio para a gestão do conhecimento. 3. Adaptação da empresa normal às tecnologias da informação e a comunicação, para apoiar processos de gestão do conhecimento; 4 . Gestão administrativa. Permanente processo de adaptação; 5. A maneira de síntese (para a discussão); 6. Conclusões, Bibliografia. 
La organización y su adaptación a las tecnologías de la información y la comunicación en procesos de gestión del conocimiento

\section{INTRODUCCIÓN}

Desde los años sesenta del siglo pasado, se habla de los cambios que las nuevas tecnologías generan en la empresa y las diferentes respuestas que esta debe tener para adaptarse a ellas. Con la introducción de la teoría general de sistemas (TGS) de Bertalanffy (1989) a la teoría administrativa y organizacional, por parte de Katz y Kahn (1978), y del enfoque de contingencia de Woodward (1965), se entiende la organización como un sistema abierto en permanente proceso de cambio y adaptación al entorno, ni mejor ni peor, solo diferente en términos de tecnología, ambiente y tamaño. Surgen así diversos enfoques gerenciales que contribuyen a la evolución y al fortalecimiento del conocimiento y al desarrollo de diversas herramientas relacionadas con la definición, la implementación, el monitoreo y el ajuste de la estrategia en la organización (Lafaurie, 2003, Murillo y otros, 2008b, 2008a, Dávila, 2001).

Parte de esa adaptación se puede lograr a través de la implementación de diversas herramientas que permitan la interacción en tiempo real con los diferentes actores vinculados a la organización. Así, la toma de decisiones, la producción, el mercadeo, el servicio al cliente, la logística, las compras, entre otras actividades, tareas, procesos y funciones de la organización, pueden estar soportados en herramientas que faciliten, agilicen y garanticen el logro de los objetivos propuestos, herramientas articuladas a políticas que dirijan las acciones organizacionales y soportadas en estrategias que viabilicen el crecimiento y la sostenibilidad de la empresa en el tiempo, en otras palabras, que generen valor y permitan su diferenciación en el mercado.

El análisis reflexivo propuesto en este artículo gira en torno a comprender la dificultad que tienen las organizaciones para articular su gestión (estrategia, estructura y cultura), a las tecnologías de la información y la comunicación (TIC), en la implementación de proyectos de gestión del conocimiento (GC). En especial porque la implementación de estos proyectos, articulados a las TIC, supone estructuras flexibles, dispuestas al cambio, con estrategias de corto y largo plazo que soporten al tiempo, la obtención de utilidades y la supervivencia de la organización; con sistemas de información que garanticen la trazabilidad de la misma como insumo necesario para la toma de decisiones y la inteligencia empresarial, siempre en ambientes de colaboración, cooperación y asociación que faciliten la transferencia de información y de conocimiento, en otras palabras, los procesos de la gestión del conocimiento y de la información.

Así, mediante un análisis reflexivo de los impactos de la implementación de proyectos de gestión del conocimiento en algunas empresas de Medellín, es posible afirmar que, contrario a lo propuesto en el párrafo anterior, las empresas implementan acciones de gestión del conocimiento y tecnologías de información 
y comunicación en estructuras altamente burocráticas, con estilos de dirección behavioristas y estrategias a corto plazo.

En síntesis, esta reflexión propone caminos de análisis en lo económico/social, para entender cómo estas herramientas, en ausencia de proyectos de transformación organizacional, no son suficientes para garantizar logros, en el corto y largo plazo. Igualmente, pone en duda la implementación de tecnología en la empresa, cuando esta no responde a estrategias, políticas, objetivos y acciones, que permeen la estructura y la cultura de la organización. En otras palabras, reconoce la importancia de las tecnologías de la información y la comunicación en los procesos organizacionales, reconoce las dificultades humanas y técnicas presentes en su implementación y, finalmente, la necesidad de que su implementación esté soportada en estrategias y políticas de cambio organizacional.

Así, para comprender estas afirmaciones, es necesario, en un primer momento, entender qué es una empresa normal con administración tradicional, la vigencia de este modelo en las empresas de hoy y las condiciones de adaptación que el mismo ofrece, ante los cambios del entorno. En un segundo momento, se presenta una aproximación a las discusiones que algunos autores realizan hoy alrededor de las TIC y su importancia en las empresas, en especial, asociada a la implementación de proyectos de gestión del conocimiento GC. Finalmente se construye una reflexión sobre las dificultades presentes en la adaptación de la estructura, la estrategia y la cultura organizacional, a las tecnologías de la información y la comunicación TIC, como herramienta necesaria para apoyar procesos de gestión del conocimiento en las empresas.

\section{LA EMPRESA NORMAL Y LA ADMINISTRACIÓN TRADICIONAL}

Para el desarrollo de este artículo, siguiendo a Patrus (2004, p.59), se entenderá por empresa normal aquella en la que los procesos son rutinas que ya no precisan ser explicadas, pues se encuentran legitimadas por el paradigma dominante. Igualmente, se entiende por administración tradicional, aquella que corresponde al conjunto de las teorías administrativas anteriores a las corrientes de cultura organizacional y de calidad total, las cuales tienen como referentes teóricos el sistema elaborado por A. Smith (1983), la organización del trabajo de Taylor (1961), la organización de las tareas administrativas y el principio de la dirección desarrollados por Fayol (1961) y Weber (1964), el trabajo de Mayo (1945) sobre los engranajes sociales (la organización informal) y el movimiento de las relaciones humanas (Aktouf, 2009; Murillo, y otros, 2008a; Zapata, Murillo, y Martínez, 2006; Dávila, 2001; Morgan, 1998). De donde se entiende que la administración tradicional es el resultado de la 
La organización y su adaptación a las tecnologías de la información y la comunicación en procesos de gestión del conocimiento

sistematización de diferentes prácticas utilizadas por los directivos para conducir los negocios, y de las justificaciones que legitimaron dichas prácticas, basadas en herramientas, principios y teorías tomados de diferentes ciencias o conocimientos con pretensiones científicas.

Esta sistematización no es producto de la reflexión de los directivos alrededor de las actividades que desarrollaban o de investigaciones académicas que buscaban la transformación de dichas actividades para la construcción de una teoría integrada. Más bien, los supuestos filosóficos de la empresa normal, de los que hablan Patrus (2004) y Aktouf (2009), surgen de la finalidad económica para el logro de los beneficios, donde la administración cumple un papel enfocado al cumplimiento de una serie de actividades integradas e interdependientes, destinadas a permitir cierta combinación de medios (financieros, humanos, tecnológicos y técnicos), para la producción de bienes y/o servicios con una finalidad lucrativa, rentable.

Esta concepción de organización tiene una base economicista, construida sobre un supuesto pragmático, que ha sido impuesto por la experiencia en la historia de la empresa, donde la finalidad económica no es algo que se presupone, sino algo que se observa en la práctica normal de las empresas. Patrus (2004) explica esta finalidad a partir de dos argumentos: el primero, entender que la finalidad económica de la empresa es un concepto a priori, anterior a la existencia de la empresa, donde la idea de concebir una empresa privada para lograr beneficios, en una economía capitalista, es anterior a su apertura; y el segundo, entender que cuando una empresa no tiene fines de ganancia en la producción de bienes y servicios, alude en términos generales, a una organización de la sociedad civil. Como las organizaciones productivas y comerciales, de iniciativa privada, tienen como finalidad alcanzar beneficios, la empresa normal hace referencia a empresas que, por definición, buscan la generación de beneficios.

Así, el objetivo de conseguir beneficios orienta la concepción de la empresa normal, su modo de funcionamiento y su administración; en otras palabras, determina su estructura, su estrategia y su cultura. En este sentido, desde una lógica económica, es normal -racional, afirma Patrus (2004) - que la empresa busque el máximo beneficio. Bajo esta premisa, la búsqueda del máximo beneficio, orientaron su trabajo los autores de la administración tradicional, preocupados por la productividad, la organización racional del trabajo, el estudio de tiempos y movimientos, la división del trabajo, los patrones de máquinas, herramientas y procesos, el planeamiento y control, el ahorro de esfuerzo, la disciplina y la autoridad (Taylor, 1961; Fayol, 1961; Weber, 1964; Mayo, 1945). Queda claro, entonces, como afirma Patrus (2004, p.57), que el estudio de la empresa normal está encaminado a lograr la perfección en el 
proceso productivo; de donde se podría inferir que el objetivo de la ciencia normal de la administración ha sido la maximización de los beneficios.

Desde esta perspectiva, la organización con finalidad económica ha tenido varias concepciones en las teorías de la organización y la gestión. La primera es el modelo mecanicista de la empresa que, desde un pensamiento racional, enfatiza dos características estructurales que distinguen las organizaciones formales de otro tipo de colectividades: los objetivos específicos claramente definidos y la formalización, en un grado alto. Las definiciones clásicas dadas por Barnard $(1938,1948)$ y por Etzioni (1964) permiten ver cómo esta perspectiva se construye sobre la noción de estructura normativa, formal de la organización, la cual puede ser deducida desde los trabajos de Taylor (1961), Fayol (1961) y Weber (1964). En este sentido, afirma Morgan (1998) que la metáfora que mejor representa la organización como sistema racional es la máquina, donde las personas no son más que partes de un engranaje, al servicio de la organización. Este modelo tiene una racionalidad instrumental cuya finalidad es la maximización de los beneficios, donde la toma de decisiones se realiza con base en criterios técnicos, soportados en normas de carácter funcional. Desde esta acepción racional, como afirman Aktouf (2009), Morgan (1998), y Murillo y otros (2008a), donde la persona solo tiene valor como medio de producción, la motivación se reduce a la búsqueda de recompensas externas o de necesidades contingentes.

El modelo de sistema natural (modelo psicosociológico) entra a escena con el surgimiento de la Escuela de las Relaciones Humanas. Los aportes de Mayo (1945), Barnard (1938, 1948), Selznick (1957), Perrow (1972) y Parsons (1968), permiten ver la organización como colectividades sociales, desde una versión de consenso social, que presupone la existencia de comportamientos cooperativos, normas y valores compartidos, para el logro de una estabilidad organizacional. Por otro lado, las teorías sociológicas del conflicto social con los aportes de Marx (1985), Simmel (1977), Dahrendorf (1979) y Giddens $(1977,1979)$ entienden el orden social como el resultado de la supresión de algunos intereses sobre otros, no desde el consenso, sino desde la imposición, la coerción y la dominación de grupos más poderosos. Finalmente, Spencer (1882), Katz y Kahn (1978), McGregor (1957) y Miller y Rice (1967), teóricos del sistema natural, aceptan la existencia de estructuras formalizadas en las organizaciones, y cuestionan su importancia y su impacto en el comportamiento de los actores sociales.

Si bien estas concepciones organizacionales emergentes no abandonan el supuesto de maximización de utilidades, incluyen en su centro de atención las relaciones humanas, dando lugar a la emergencia de conceptos como la solidaridad, la cooperación, la participación y la descentralización. De esta manera introducen la 
La organización y su adaptación a las tecnologías de la información y la comunicación en procesos de gestión del conocimiento

dimensión psicosocial de la organización, donde se reconoce un individuo dotado de libertad y de voluntad, y admiten que las motivaciones humanas necesitan algo más que la búsqueda de recompensas externas.

En suma, como afirman Patrus (2004), Aktouf (2009) y Murillo y otros (2008a), a pesar de que conservan su carácter funcionalista, estas nuevas acepciones organizacionales, al concebir la organización como un sistema abierto, son más sensibles a su relación con el entorno y a las demandas de los organismos civiles por la responsabilidad social y ambiental. No aspiran a la afirmación de la persona humana, sino a la supervivencia de la organización por medio de la adaptación al medio. De ahí que esta mirada organizacional se configure como un avance importante en relación con el anterior.

\section{TECNOLOGÍAS DE LA INFORMACIÓN Y LA COMUNICACIÓN: HERRAMIENTAS DE APOYO PARA LA GESTIÓN DEL CONOCIMIENTO}

Algunos estudios han abordado el papel de las tecnologías de la información (TI) en la gestión del conocimiento (GC). Autores como Alavi y Leidner (2001), Markus (2001), Chang, Sangjae y Won (2005), Meroño (2005), López-Nicolas y Meroño (2009), citados en Rueda (2012, p. 82), se refieren a sistemas basados en tecnologías modernas: Internet, intranet, navegadores, almacenes de datos y agentes de software para sistematizar, facilitar y agilizar la GC en la organización.

En el modelo propuesto por Tissen, Andriessen y Lekane (2000), citado en Rueda (2012, p. 82), la gestión del conocimiento, GC, debe trabajarse desde dos miradas de análisis: una estratégica y una operativa. La GC estratégica permite la creación de estructuras y procesos que fomenten el conocimiento, para profesionales de conocimiento. Por su parte, en la GC operativa, participan las TIC, como soporte para organizar, clasificar y distribuir el conocimiento desde y hacia los colaboradores de la organización.

El modelo propuesto por Kerschberg y Weishar (2002), citado en Rueda (2012, p. 83), integra el conocimiento en una organización a través de las TIC, en tres niveles, con diferentes fuentes de conocimiento a las que se puede tener acceso. El primer nivel es la caja de fuentes, fuentes externas y repositorios: web, e-mail, de texto, de medios y de dominios. El segundo nivel, la capa de GC, tiene el repositorio del conocimiento, que soporta el proceso de GC: adquisición, refinamiento, almacenamiento, distribución y presentación. En el tercer nivel, la capa de presentación, se encuentra el portal de conocimientos, donde los trabajadores comunican, colaboran y comparten conocimiento. 
Skyrme (2002), citado por Rueda (2012, p. 83), incluye, en el modelo de Kerschberg y Weishar (2002), las TIC como parte de la infraestructura del conocimiento, a través de cuatro niveles de exigencia: conexión, comunicación, conversación y colaboración. Así el modelo tendría un primer nivel de capa de fuentes de datos, un segundo nivel de repositorio de conocimiento donde se ubican los servicios de data mining, servicios de tagging de metadatos, ontología-tesauro inteligente, servicios de actualización de conocimiento, workflow, data warehouse, servicios de unión, servicios de agentes, servicios intermediario y otros servicios intermediario. Finalmente se encuentra la capa de GC, configurada por el portal de conocimiento y servicios de búsquedas, mensajes y colaboración, videoconferencia, grupos de discusión y creación de conocimientos.

A partir de los modelos de Tissen, Andriessen y Lekane (2000), Kerschberg y Weishar (2002) y los aportes de Skyrme (2002), Del Moral y otros (2007), citado en Rueda (2012, p. 85), presentan un modelo soportado en tres aspectos fundamentales: estructura, función y comportamiento.

La estructura contiene tres niveles. a) El nivel de objetos o de información hace referencia a las fuentes de información: bases de datos, documentos escritos, correo electrónico, web, etc., y a la infraestructura tecnológica que soporta la información: intranet, Internet, procesadores de textos, software, multimedia. b) El nivel de contenidos de conocimientos se refiere a la ingeniería del conocimiento que provee técnicas y herramientas para formar repositorios de conocimientos que permiten extraer, recopilar y almacenar las distintas representaciones y formatos en los que se consolide el conocimiento, para que sea de utilidad para las personas que lo requieran. c) El nivel de presentación y comunicación se refiere a la interfaz de los usuarios: videoconferencias, mensajes, grupos de práctica y portales que permiten al usuario tener acceso al conocimiento.

La función se basa en la integración (Pull) y la conectividad (Push) de los datos almacenados, encargadas de entregar la información solicitada por los usuarios y enviar la información a las personas relacionadas con la toma de decisiones en sus áreas de trabajo.

Finalmente, el comportamiento hace referencia a la organización de las acciones.

En síntesis, para Meroño (2005), citado por Rueda (2012, p. 87-88), las TI generan un fuerte impacto en los procesos de GC en las empresas y en su relación con el entorno. Internet, groupware, repositorios de conocimiento, sistemas de información, mapas de conocimiento, portales de conocimiento configuran hoy alternativas de acceso a TI para las empresas. Igualmente existen diferentes categorías de software 
La organización y su adaptación a las tecnologías de la información y la comunicación en procesos de gestión del conocimiento

para soportar los procesos de conversión de conocimiento: combinación (sistemas basados en intranet, gestión de documentos electrónicos, inteligencia de negocio, herramientas de inteligencia competitiva), externalización (groupware, workflow, sistemas basados en conocimiento), socialización (mapas de conocimiento), internacionalización (herramientas de apoyo a la innovación), socialización, externalización y combinación (portales de conocimiento).

\section{ADAPTACIÓN DE LA EMPRESA NORMAL A LAS TECNOLOGÍAS DE LA INFORMACIÓN Y LA COMUNICACIÓN, PARA APOYAR PROCESOS DE GESTIÓN DEL CONOCIMIENTO}

Con la implementación de proyectos de GC en la empresa y el uso de herramientas TIC para soportarlos, la empresa normal ha experimentado algunos cambios en la estructura de la organización del trabajo, dejando de lado el énfasis sobre la capacidad física del trabajador (Taylor, 1961) hacia su conocimiento (Drucker, 1993, 1994; Nonaka y Takeuchi, 1995, 1999; Nonaka y Toyama, 2003).

Emergen modelos organizacionales más flexibles, dinámicos y complejos, modelos matriciales, estructuras orientadas a proyectos, organizaciones adhocráticas (Mintzberg y Mchugh, 1985), equipos multidisciplinares, organizaciones planas (Carnoy, 2001), organizaciones en red. Estos modelos generan cambios en los principios del trabajo, miradas menos jerárquicas, más horizontales, orientadas a procesos, al capital intelectual (Kaplan y Norton, 2000; Edvinsson, 1993; Edvinsson y Malone, 1997; Brooking y Motta, 1996; Sveiby, 1997; Euroforum, 1998; Bueno, 1998; Camisón y Palacios, 1999; CIC-IADE, 2003) y al fomento del capital intangible (Mabilon, 2010).

El cambio aquí mencionado no se reduce a la organización interna: afecta a todos los actores que se encuentran alrededor de la misma. Surgen, de esta manera, propuestas para fomentar un marco empresarial colaborativo, globalizado, con mayor presencia de actividades subcontratadas y externalizadas que dan lugar a etiquetas tales como sociedad de la información y del conocimiento, economía digital, e-business, empresa en red, economía del conocimiento, economía global, web 2.0, redes sociales, capital relacional, capital intangible, empresa 2.0, empresa extendida y gestión del conocimiento 2.0, entre otros.

Como sucedió con la calidad y con los procesos, la tecnología y el conocimiento, dejan de ser modas administrativas para convertirse en procesos, funciones, actividades normales de la organización. Como afirma Mabilon (2010), emerge un modelo de organización productiva que superar la cadena integral y secuencial de operaciones, una organización con procesos colaborativos, horizontalidad, flexibilidad, información, relaciones y procesos extendidos, conocimiento cooperativo y fomento del capital humano. Surge la empresa-red, denominada así por Castells 
(2003), Mintzberg y Heyden (2006) y Tapscott (2006), una organización que genera y aprende de su conocimiento (Drucker y otros, 2003).

Con el reconocimiento de la empresa-red, surgen conceptos como flexibilidad, autonomía, fomento de recursos y capacidades distintivas, foco en el centro del negocio, recursos intangibles y capital intelectual. Esto fomenta la desagregación y externalización de actividades y procesos, soportados en nuevas tecnologías de la comunicación globales y en sistemas de información $24 * 7$, que impulsan la globalización de la producción y una creciente sofisticación de las operaciones.

Con la desregularización del mercado y de la empresa, emergen nuevas lógicas de prestación de servicios (laborales), que permiten la libre movilidad de bienes, servicios, materiales, capitales y personas, tales como Outsourcing-offshoring (externalización-deslocalización), offshoring (deslocalización), outsourcing (externalización) y bodyshopping (compra de cuerpos), entre otras. Con esta desregularización surge la deslocalización empresarial, la pérdida de competitividad industrial, la dispersión del conocimiento o los excesos de los mercados financieros. En contraste, la empresa-red ofrece una fuente de ventaja competitiva vinculada a la transformación empresarial a través del uso de la información y el conocimiento, que no se limita a un cambio organizacional o a la inversión en TIC, pues configura una compleja red de activos humanos y materiales -tangibles e intangibles- orientados a maximizar el valor a través de la información y del conocimiento.

En otras palabras, con la evolución de las TIC, no solo se da un gran desarrollo en términos de hardware y de software que se convierte en verdaderas plataformas que facilitan y potencian ambientes colaborativos, sino que se viabiliza una de las condiciones más importantes de este proceso de cambio: generar ambientes donde los trabajadores del conocimiento, de los que habla Drucker (1993), estén más proactivos frente a la posibilidad de compartir el conocimiento, en la medida en que salen de sus espacios de conocimiento individual y crean redes de conocimiento que, extrapoladas a la comunidad, generan todo un supra-sistema de comunicaciones y de nuevo conocimiento para la ventaja mutua.

El desarrollo de las TIC permite la construcción de plataformas que van más allá del intercambio de datos, información o documentos. Estas plataformas de información permiten soportar todo un sistema de colaboración, donde se viabiliza el proceso de creación, transformación, almacenamiento, uso y distribución del conocimiento. Sin embargo, es importante comprender, como afirma Bhatt (2000), que en los procesos de GC, la tecnología soporta el 10\% de todo el proceso, mientras que el $70 \%$ está soportado en las personas y un $20 \%$ en los procesos. En otras 
La organización y su adaptación a las tecnologías de la información y la comunicación en procesos de gestión del conocimiento

palabras, los procesos de GC estarán soportados sobre la estrategia, la estructura y la cultura de la organización.

\section{GESTIÓN ADMINISTRATIVA. PERMANENTE ADAPTACIÓN}

\subsection{La estrategia}

En sus publicaciones, Prahalad (1994, 2004, 2006), Prahalad y Krishnan (2007), Prahalad y Hamel (1990, 1994, 1998), Prahalad y Gibson (1997), dejaron como legado siete nuevas visiones sobre la estrategia: (1) la esencia de la estrategia no es la gestión de los recursos actuales de la organización sino la gestión del futuro, crear el futuro, no desde la reingeniería de procesos sino desde la regeneración de las estrategias centrales; (2) ese competir en el futuro significa mantener una continuidad que asegure que la empresa siempre introduzca nuevos beneficios (una difícil relación entre la ética del beneficio cortoplacista y la continuidad de la corporación); (3) más que planificar se deben potenciar las competencias internas (ínter-empresariales) de las organizaciones; (4) esa visión de futuro se logra a través de un proceso de síntesis, no de análisis (integración, no separación); (5) con su enfoque en la base de la pirámide muestra que las grandes corporaciones no solo pueden ganar dinero al vender en los países más pobres, sino contribuir a mejorar las condiciones de vida de estos países, lo que permite disminuir la brecha con los países ricos; (6) propone revisar el paradigma del management y de la estrategia, (7) tiene el coraje de romper las ataduras académicas mediante la crítica de las escuelas y sus enfoques reduccionistas. Así, definiciones como core competences (competencias centrales) de Prahalad y Hamel (1990), la estrategia de la co-creación de valor, el concepto de prosumidor, y el de la base de la pirámide revolucionaron el mundo de los negocios basados en la tecnología.

En el Software 2006 Conference, Prahalad (2006) explica cómo los centros tradicionales de innovación (tipo Silicon Valley) pronto serán nodos independientes en un ecosistema tecnológico global. No solo se asiste a la deslocalización de las organizaciones, afirma el autor, sino a la desaparición de las barreras entre las industrias verticales; pronto se observará cómo toma impulso la convergencia industrial. Convergencia que trabaja sobre cuatro tópicos de discusión: (1) los ecosistemas basados en locaciones específicas, (2) la emergencia de ecosistemas globales, (3) la emergencia de ecosistemas complejos que promueven la convergencia industrial y (4) ecosistemas que incluyen para sí la mayor parte de la población mundial.

Para Prahalad $(2004,2006)$ la visión tradicional de la creación de valor ha estado centrada en la empresa y deja de lado la percepción de valor que los consumidores tienen. Sin embargo, con el advenimiento de las TIC, la empresa está abocada a ser 
más sensible sobre la experiencia del usuario. Así, junto con Ramaswamy (Prahalad y Ramaswamy, 2004a, 2004b, 2005) propone la creación de valor en sintonía con los clientes, una co-creación de valor que satisfaga a las partes implicadas. Co-creación que comprende cuatro elementos de intercambio que determinan la experiencia: el manejo de la transacción, cómo se establece la selección, la experiencia del consumo y la relación precio-desempeño. Co-creación construida sobre cuatro bloques: diálogo, acceso, reducción del riesgo y transparencia. En otras palabras, en Co-creación, los autores proponen que el origen de la innovación no se limita a la empresa; los clientes, proveedores y otros agentes externos pueden convertirse en fuentes de innovación, lo que conduce a la empresa al concepto de innovación abierta.

En relación con la continuidad de las compañías, Prahalad (Prahalad y Ramaswamy, 2004b, 2005; Prahalad y Gibson, 1997; Prahalad y Hamel, 1994) asegura que su logro depende de la rentabilidad de las mismas. Para el autor, crecer y cambiar es un criterio esencial para la competitividad; la estabilidad está mediada por el proceso permanente de adaptación de la compañía con su entorno. Se trata de un proceso de evolución permanente en medio de la continuidad, donde la continuidad es entendida como la capacidad de aceptar el cambio y de saberlo manejar. No se trata de una estabilidad que frena y estanca, sino de un proceso de adaptación permanente al entorno: medio, mercados, tecnología, productos y clientes. Se trata de un equilibrio entre el corto plazo y la eficiencia operativa, junto a una perspectiva estratégica -y prospectiva- de largo plazo; de la coherencia entre la asignación de recursos a largo plazo y el logro de eficiencia a corto plazo, para la acumulación y distribución de los recursos.

Para el autor no existe una relación directa entre competitividad y eficacia de capital con localización (país de origen). Existe un conjunto de tareas empresariales comunes que se realizan con corrección. Las compañías transnacionales con ubicación en diferentes países y culturas necesitan un marco global que aúne los esfuerzos de personas de origen, historia y filiación diferentes. En otras palabras, para Prahalad y Krishnan (2007) y Prahalad y Ramaswamy (2005), una empresa no puede denominarse a sí misma mundial si no es capaz de crear un marco intelectual que permita a las personas de diferentes países relacionarse a través de ella.

En contraste con el posicionamiento estratégico de Porter (1980, 1985, 1998), diferenciación, costes o focalización y sus cinco fuerzas competitivas, Prahalad y Hamel $(1994,1998)$ reivindican las habilidades y competencias distintivas, al constatar que las ventajas competitivas no dependen del entorno o de la industria, sino de la capacidad de la empresa de conseguir esas ventajas en su interior. Así, estos autores, en su libro Compitiendo por el futuro (Prahalad y Hamel, 1996), al evidenciar el 
La organización y su adaptación a las tecnologías de la información y la comunicación en procesos de gestión del conocimiento

posicionamiento estratégico en relación con las competencias distintivas, permiten el resurgimiento de un viejo debate (sesentas) entre estrategia y estructura.

\subsection{La estructura}

Si bien el debate entre estrategia y estructura que surgió en los años sesenta se ha superado, el nuevo debate, hoy, gira alrededor de las configuraciones organizacionales de la empresa-red. Así, los temas de regionalización, encadenamiento, integración vertical u horizontal se encuentran en torno a conceptos como asociatividad y cooperación entre empresas, y entre redes de empresas, siempre alrededor de un objetivo fundamental, la gestión de los procesos de conocimiento: creación, transformación, almacenamiento, distribución y uso.

Con la propuesta de Aktouf (2009), de la administración renovada, surge una serie de ideas que, de una u otra manera, alteran la forma en que se entendían la organización y su administración. Con la gestión por proyectos y los desarrollos de las TIC, se hace necesario mejorar la capacidad como se enfrenta la creciente complejidad de los ambientes organizacionales. Surgen así estructuras y formas de gestión, denominadas por Mintzberg (2001) matriciales, transversales y de redes. A medida que crece la complejidad del proyecto y su alcance, afirma Zapata (2008), es recomendable pasar a una forma de administración que supere las nociones tradicionales de estructura: funcional, por departamentos, por productos. Se requiere una administración matricial o transversal, es decir, la introducción de un funcionamiento transversal a la ya conocida configuración vertical. En palabras de Aktouf (2009, p. 655), "se trata de hacer salir las relaciones interpersonales de la organización de sus habituales fronteras jerárquicas, funcionales, departamentales, para hacerlas estallar por múltiples pasarelas trans-estructurales que agregan a las relaciones tradicionales verticales nuevas relaciones, más directas, horizontales y diagonales".

Estos cambios estructurales son necesarios para el buen funcionamiento de los proyectos, que no están en capacidad de soportar los plazos y la tramitomanía de una estructura jerárquica y funcional. Requiere más bien, como afirma Aktouf (2009, p. 655) de "una capacidad de contactos y de acciones en tiempo real, al cual se acomoda, con frecuencia, muy mal el "espíritu piramidal" concebido para la centralización, el control, el orden, lo previsto-previsible, el no-ruido y el tiempo diferido".

Las nuevas tendencias en la gestión replantean la ventaja o no de mantener los esquemas tradicionales de la administración de la empresa normal que trabaja con procesos lineales. Desde la década de 1970 se ha hecho el llamado hacia estructuras más flexibles, menos robustas y jerárquicas, sin tanta tramitología y excesos de poder, que favorezcan la adaptación al cambio. Con la gestión del conocimiento, 
soportada en TIC, las propuestas de flexibilidad adquieren mayor relevancia, en especial, porque la generación de valor, en procesos de GC, incluye cambios significativos en la estructura de la organización: el poder, la toma de decisiones, el acceso a la información y las formas de comunicación, entre las más importantes.

\subsection{La cultura}

Si bien desde los años cuarenta se ha hablado en la administración de la importancia de los símbolos y del lenguaje para comprender la dinámica de los grupos, es en los ochenta cuando se empieza a hablar seriamente de la necesidad de tener una buena cultura en la organización (Ouchi, 1981; Peters y Waterman, 1982). No obstante, como afirma Aktouf (2009, p. 660), se sigue dando atención a las virtudes de la administración científica. Prueba de ello es la importancia que adquieren los Sistemas de Gestión de la Información (Management Information Systems), en la década de 1980, con la re-edición de The New Science of Management Decision de Simon (1977), dando lugar a la presencia de la gestión digital con computadores, programas y sofisticados algoritmos.

Esta tendencia olvida aportes de autores como Herzberg (1980, p. 70), quien afirmara que "no podemos dirigir a la gente científicamente" (we cannot manage people scientifically). Sin embargo, en la gestión por proyectos, bajo la dirección de ingenieros y economistas, domina el escenario organizacional la lógica técnico-financiera. De hecho, a pesar de las denuncias realizadas por Peters y Waterman (1982), a los excesos de racionalismo y de instrumentalismo presentes en la gestión hasta los años sesenta, en la gestión por proyectos, incluso en sus facetas más humanas, afirma Aktouf (2009, p. 660), la gestión se mira como "el baluarte más tecnificado y el más resistente del hard management". En este mismo sentido, el autor (Aktouf, 2009, p. 661-662) afirma que:

Si hay una lección para aprender es que ningún símbolo, ningún sistema de representaciones colectivas, ninguna cultura podría ser "creada", inculcada o impuesta sin una base en condiciones de vida concretas y materiales. Pero como el proyecto es, por definición, no permanente, heterogéneo, móvil, con frecuencia ínter-regional y hasta internacional, ¿̇perderán, acaso, importancia las preguntas sobre cultura y símbolos? Es precisamente ahí donde las nociones de "visiones compartidas" y de coherencia entre los "recursos-representaciones y la vida material concreta" adquieren toda su importancia. El contexto de proyecto, lejos de estar fuera del alcance de los fenómenos de "culturas", está, por el contrario, marcado por sólidas "culturas de oficios": profesiones que elaboran sus propias maneras de ver, de actuar, de ser líderes, de vencer las angustias, profesiones que conciben sus propias ideologías, sus propios "ritos y costumbres", etc. 
La organización y su adaptación a las tecnologías de la información y la comunicación en procesos de gestión del conocimiento

\section{A MANERA DE SÍNTESIS (PARA LA DISCUSIÓN)}

Con el análisis realizado en el artículo surgen nuevos interrogantes, pues los avances de las TIC hacen que hoy en día los entornos organizacionales sean cada vez más complejos; de ahí que la gestión deba tener las herramientas, los métodos, las técnicas y el conocimiento necesarios para hacer que la organización navegue en forma adecuada por ese entorno cambiante, turbulento en ocasiones. Sostenibilidad, rentabilidad, competitividad y productividad constituyen los cuestionamientos permanentes sobre los cuales, los directivos, día a día, hacen planes, definen objetivos, fijan metas, determinan políticas, diseñan estrategias y contratan personal. Hoy se vive un proceso administrativo fortalecido, afirma Aktouf (2009), donde la planeación, la dirección, la organización y el control configuran las funciones fundamentales de la gestión y de la organización.

Entonces, ¿cuáles son las diferencias entre las propuestas de finales del siglo XIX y las de inicios del siglo XXI? Hoy la administración usa un lenguaje menos agresivo, más metafórico, diría Morgan (1998), pero al final siempre busca lo mismo: la esencia financiera y económica de la que hablaba Patrus (2004). Hace un siglo, para Taylor (1961), el énfasis se ponía sobre el hombre correcto en el puesto correcto, para labores que necesitaban más fuerza física que destreza mental, y lograr el menor costo del trabajo subdividido (Babbage, 2009). Hoy se valora la capacidad cognitiva de ese individuo en el trabajo, su conocimiento, tácito y explícito (Davenport y Prusak, 1998; Nonaka y Takeuchi, 1995).

Si bien el conocimiento individual y especializado es importante para la empresa, en aras de conservar la especialización del trabajo y del conocimiento, en una estructura organizacional que defina orden y jerarquía, ese conocimiento es relevante en la medida en que pueda ser transmitido para generar un aprendizaje colectivo y elevarlo a aprendizaje organizacional. En otras palabras, se trata de la integración colectiva de las personas y los conocimientos, alrededor de una situación problemática, que logre generar la sinergia suficiente para encontrar la solución más adecuada. En palabras de Maldonado (2011), se trata de comprender que hoy lo más importante no es encontrar respuestas, sino saber elaborar preguntas; desarrollar procesos de Inteligencia Colectiva, como afirman Martínez y Vásquez $(2011,2012)$ y Lévy (2004) o configurar clústers de conocimiento, como afirma Nieto (2007).

No obstante estos cambios, hoy se sigue discutiendo alrededor de algunas preguntas relacionadas con la gestión empresarial: ¿cómo optimizar los recursos?, ¿cómo maximizar las utilidades?, ¿cómo minimizar los costos?, ¿cómo crear valor para la empresa?, ¿cómo generar una ventaja competitiva sostenible?, ¿cómo generar valor agregado para el producto o para el servicio?, ¿cómo garantizar la diferenciación 
en el mercado el tiempo suficiente para recuperar la inversión realizada en I+D+i?, ¿cómo sacar las mayores ventajas de las oportunidades que ofrece en entorno?, ¿cómo minimizar las amenazas del entorno?, ¿cómo lograr la mayor productividad de los trabajadores?, ¿cómo hacer que los trabajadores estén satisfechos y trabajen más y mejor?, ¿cómo disminuir los costos laborales? y ¿cómo usar eficientemente los recursos escasos?, entre otras similares.

Al final, las preguntas por el cómo de la gestión se conservan en el tiempo; preguntas que determinarán el estilo de dirección en términos de la estrategia, la estructura y la cultura; preguntas que definirán la forma como la empresa se adapta al cambio. Lo que no debería suceder es que las respuestas a estas preguntas estén enmarcadas en los fundamentos de la administración tradicional.

Con las nuevas tendencias administrativas surgen nuevos conceptos que empiezan a hacer parte del argot directivo y organizacional, tales como: autonomía del personal; libertad en el ejercicio de poder y la toma de decisiones, para la resolución de problemas y la generación de nuevo conocimiento; cooperación para la creación y transformación de conocimiento; colaboración y asociatividad para la transferencia de conocimiento; confianza, para generar los espacios adecuados que fomenten esa colaboración. Estos conceptos, ligados a conocimiento, cambian la forma de ver al trabajador. Así el lenguaje administrativo migra de recurso humano a personal, a talento humano y a capital intelectual. Vuelve así, una vez más, la administración, la mirada hacia lo humano individual y colectivo, al entender al trabajador como actor y generador de conocimiento, aprendizaje, creatividad e innovación en la organización.

Al mismo tiempo, los panópticos de control de los que habla Foucault (1996), soportados en las TIC, se vuelven cada vez más sofisticados, menos agresivos y más efectivos, al punto de camuflarse en el diario quehacer de las personas. Es posible que en un primer momento generen una reacción negativa por parte de los empleados, pero poco a poco se facilitan procesos de adaptación a las nuevas tecnologías de control, hasta el punto de hacer que los trabajadores entiendan que es por su propio bien, por la seguridad de ellos, de sus puestos de trabajo y de la organización misma. El discurso de la seguridad justifica la proliferación de artefactos de control tales como: la BlackBerry, la Palm, el chip de seguridad en los carnés de identificación de los trabajadores, el GPS en el dispositivo celular y/o en el vehículo y los dispositivos cerrados de seguridad, entre otros.

Hay una esquizofrenia colectiva, un desesperado deseo de controlar o de ser controlado, donde cada individuo vende su libertad a cambio de las comodidades del mercado, como afirma Marcuse (1954). Poco a poco el control sobre el trabajo y el espacio de trabajo rompe las fronteras de lo laboral/social y empieza a avanzar 
La organización y su adaptación a las tecnologías de la información y la comunicación en procesos de gestión del conocimiento

sobre los espacios personales de los sujetos, hasta convertirse en parte de su rutina diaria; así el control que antes era visible para el trabajador, hoy se camufla en la tecnología, al punto de cambiar las percepciones de las personas sobre los efectos y las utilidades de ella. Los límites ya no existen, se han roto todas las fronteras, afirma López (2005).

Para concluir esta reflexión, queda por decir que, si bien los avances en las TIC, han generado cambios en algunos de los supuestos sobre los cuales se hacía la administración tradicional, al tiempo, han fortalecido otros presupuestos de la empresa normal. En otras palabras, coexisten la inercia frente al cambio con el dinamismo del mercado, la tecnología y la innovación; coexisten las viejas formas de administración junto a los cambios que la tecnología exige, en términos de flexibilidad del sistema, sinergia, acceso a información para la toma de decisiones y el ejercicio de un poder más democrático. Coexisten la empresa normal con administración tradicional y la empresa red en sinergia con sus elementos. Pero, por encima de todo, lo que no ha cambiado es el supuesto filosófico sobre el cual se crea la empresa normal y se condiciona la gestión, la ganancia; la ventaja es que las tecnologías sirven a este propósito fundamental.

\section{CONCLUSIONES}

Si bien los diferentes enfoques gerenciales tratan de proponer modelos, metodologías que resistan el proceso de adaptación de la empresa a las nuevas tendencias del entorno, para soportar respuestas en tiempo real que garanticen niveles de competitividad, también es cierto que muchos de estos modelos o enfoques se soportan en herramientas (TIC) para agilizar, soportar procesos, generar indicadores de productividad, de medición del desempeño, entre otros, que faciliten la adaptación de la empresa para su supervivencia y crecimiento. No obstante, los modelos apoyados en herramientas (TIC) no constituyen garantía de éxito en términos de productividad, competitividad y generación de valor.

Los autores analizados por Rueda (2012), en su tesis doctoral, permiten comprender la importancia de las TIC, como herramientas que soportan la operación de proyectos de GC en la empresa. En otras palabras, enfatizan en la importancia de las TIC como plataformas que soportan la toma de decisiones, la gestión de las formas del conocimiento y los procesos de adaptación de la organización al entorno.

Sin embargo, es importante recordar que las TIC constituyen apenas un 10\% de todo el proceso de GC en una empresa. Es posible que en ausencia de ellas los resultados no puedan ser alcanzados en el tiempo y en la forma propuesta por la empresa, pero su existencia no garantiza el logro de los resultados que se espera 
obtener. El componente humano es el factor determinante del éxito o fracaso de este tipo de propuestas en la empresa. Un proyecto de GC ligado a una estrategia, definido y orientado por políticas de la empresa, con estrategias y objetivos claros y alcanzables, con procesos bien establecidos, con estructuras flexibles que permitan la libre movilidad de información y de conocimiento para la creación, transformación, almacenamiento, distribución y uso del conocimiento, y soportado en TIC, tiene mejores oportunidades de implementación y logro de resultados.

La adaptación de la estructura, de la estrategia y de la cultura a los cambios propuestos por el proyecto de gestión del conocimiento en la empresa, soportado en tecnologías de información y comunicación, podrá no ser la fórmula de éxito empresarial, pero garantiza, en la medida de lo posible, un mejor desempeño de lo humano, lo tecnológico, lo financiero y los procesos, y de la organización misma.

\section{BIBLIOGRAFÍA}

Aktouf, Omar (2009). La administración, entre tradición y renovación. Cali, Editorial Universidad del Valle, Gaëtan Morin Éditeur, $4^{a}$ edición en español, 680p.

Alavi, M y Leidner, D (2001). Review: Knowledge Management and Knowledge Management Systems: Conceptual Foundation and Research Issues. MIS Quarterly, Vol. 25, N. ${ }^{\circ} 1$, pp. 107-136.

Babbage, Charles (2009). On the economy of machinery and manufactures. En: Omar Aktouf (2009). Administración y organizaciones. Entre la tradición y renovación. Editorial Universidad del Valle.

Barnard, Chester (1938). The Functions of the Executive. Cambridge, MA, Harvard University Press. Thirtieth Anniversary Edition, 248p.

Barnard, Chester (1948). Organization and Management, Selected Papers. Cambridge, MA, Harvard University Press, 240p.

Bertalanffy, Ludwig von (1989). Teoría General de los Sistemas. México: Fondo de Cultura Económica, 168p.

Bhatt, Ganesh (2000). Organizing knowledge in the knowledge development cycle. En: Journal of Knowledge Management, Vol. 4, N. ${ }^{\circ}$, pp.15-26.

Brooking, A., y Motta, E. (1996). Taxonomy of Intellectual Capital and a Methodology for Auditing it. En: 17th Annual Business Conference, McMaster University, Hamilton, Ontario, Canada, January 24-26.

Bueno, E. (1998). El capital intangible como clave estratégica en la competencia actual. En: Boletín de Estudios Económicos, Vol. LIII, N. ․ 164, pp. 205-229. 
La organización y su adaptación a las tecnologías de la información y la comunicación en procesos de gestión del conocimiento

Camisón, Carlos, y Palacios, Diego. (1999). Un modelo para la medición del capital intelectual en la empresa: el modelo Nova. [En líneal Disponible en www.gestiondelconocimiento. com [marzo de 2010].

Carnoy, Martín. (2001). El trabajo flexible en la era de la información. Madrid, Alianza Editorial, 290p.

Castells, Manuel. (2003). La interacció entre les tecnologies de la informació i la comunicació i la societat xarxa, un procés de canvi històric. Coneixement i societat, En: Revista d'Universitats, Recerca i Societat de la Informació, 1r. quadrimestre, pp. 8-21.

Chang Lee, K., Sangjae, L., y Won Kang, I. (2005). KMPI: measuring knowledge management performance. En: Information y Management, Vol. 42, pp. 469-482.

CIC-IADE. (2003). El modelo "Intellectus" a examen: Nuevos desafíos. En: Documentos Intellectus N. ${ }^{\circ}$ 6. Madrid: Instituto Universitario de Administración de Empresas (IADE) y Universidad Autónoma de Madrid.

Dahrendorf, Ralf (1979). Las clases sociales y su conflicto en la sociedad industrial. Madrid, Ediciones Rialp, 220p.

Davenport, Thomas H., y Lawrence Prusak (1998). Working Knowledge, How Organizations Manage What They Know. Cambridge, MA, Harvard Business School Press, 220p.

Dávila, Carlos (2001) Teorías organizacionales y administración. Bogotá, Interamericana, 290p.

Del Moral Bueno, Anselmo; Pazos, Juan; Rodríguez, Esteban; Rodríguez, Alfonso y Suárez, Sonia (2007). Gestión del Conocimiento. España: Thomson Editores, 499p.

Drucker, Peter (1993). El management del futuro. México, Norma, 290p.

Drucker, Peter (1994). Post-capitalist society. New York, Harper-Business, 320p.

Drucker, Peter; Nonaka, Ikujiro; Argyris, Chris; Brown, James Scott y Garvin, David (2003). Gestión del conocimiento, España, Ediciones Deusto, Harvard Deusto Business Review. 245p.

Edvinsson, Leif (1993). Intellectual Capital shapes the Futures Enterprise. En: Scandinavian Insurance Quarterly, N. ${ }^{\circ}$.

Edvinsson, Leif y Malone, Michael (1997). Intellectual capital, realizing your company's true value by finding its Hidden Brainpower. New York, Harper-Business, 225p.

Etzioni, Amitai (1964). Modern Organizations. Englewood Cliffs, New Jersey, Prentice-Hall, $248 p$.

Euroforum (1998). Medición del Capital Intelectual. Modelo Intelect, Euroforum, Madrid. [En líneal Disponible en http://gestiondelconocimiento.com/modelo_modelo_ intelec. htm [mayo de 2009]. 
Fayol, Henri (1961). Administración industrial y general. México, Herrera Hermanos, 120p.

Foucault, Michael (1996) El sujeto y el poder. En: Revista de Ciencias Sociales Departamento de Sociología Facultad de Ciencias Sociales, Fundación de Cultura Universitaria, N. ${ }^{\circ}$ 12, Montevideo.

Giddens, Anthony (1977). El capitalismo y la moderna teoría social. Barcelona, Labor, 82 p.

Giddens, Anthony (1979). La estructura de clases en las sociedades avanzadas. Madrid, Alianza, pp. 27-35.

Herzberg, Frederick (1980). Humanities, Practical management education. En: Industry Week, Vol. 206, N. ${ }^{\circ}$, pp. 69-72.

Kaplan, Roberto y Norton, David (2000). Cuadro de Mando Integral. Barcelona, Gestión 2000, 332p.

Katz, Daniel y Kahn, Robert (1978). The social psychology of organizations. New York, John Wiley y Sons, 2a edition, 160p.

Kerschberg, Larry y Weishar, D. (2002). Conceptual models and architectures for advanced information systems. En: Applied Intelligence, Vol. 13, N. 2. PÁGINAS

Lafaurie, Marta (2003). Análisis comparativo de las herramientas estratégicas más conocidas en nuestro medio empresarial. En: Pensamiento y Gestión, N. 15, pp. 111-157.

Lévy, Pierre (2004). Inteligencia colectiva por una antropología del ciberespacio. Organización Panamericana de la Salud. Washington. BVS Biblioteca virtual em saúde, BIREME - OPS - OMS. [En líneal Disponible en http://inteligenciacolectiva.bvsalud.org lagosto de 2010].

López, Francisco (2005) Dobles sentidos y connotaciones en el discurso administrativo, dispositivos de poder en las organizaciones. En: Nuevo Pensamiento Administrativo, Fernando Cruz Kronfly (Comp.). Cali, Universidad del Valle, pp. 25-40.

López-Nicolás, C. y Meroño, A. (2009). The impact of organizational culture on the use of ICT for knowledge management. En: Electron Markets, Vol. 219, pp. 211-219.

Mabilon, August (2010). Retos de la gestión de las tecnologías de la información en una nueva era de colaboración social. En: Nuevas tendencias en Management, Fundamentos y aplicaciones. Barcelona, Bresca Editorial, S. L., pp. 97-125.

Maldonado, Carlos Eduardo. (2011). Aportes del pensamiento complejo a la administración. Director del Doctorado en Ciencias de la Dirección Universidad del Rosario. En: Conferencia de la Coordinación Académica de la Maestría y el Doctorado en Administración, Medellín, Universidad EAFIT, 26 de mayo de 2011.

Marcuse, Herbert (1954). One-dimensional man. Boston, Bacon Press, 179p. 
La organización y su adaptación a las tecnologías de la información y la comunicación en procesos de gestión del conocimiento

Markus M. (2001). Toward a theory of knowledge reuse: Types of knowledge reuse situations and factors in reuse success. En: Journal of Management Information Systems, Vol. 1, N. ${ }^{\circ} 18$, pp. 57-93.

Martínez, Jenny y Vásquez, José Alfredo (2011). Modelo de creación de inteligencia colectiva en una red de empresas. En: Memorias II Simposio Iberoamericano de Estudios Gerenciales: una mirada interdisciplinar a la innovación, Ochoa, Héctor (Coord.). Cali, Universidad ICESI.

Martínez, Jenny y Vásquez, José Alfredo (2012). Methodology for implementing a collective intelligence creation model in business networks. Ponencia presentada en la 13th European Conference on Knowledge Management, ECKM 2012, Universidad Politécnica de Cartagena, Spain, 6-7 september.

Marx, Karl (1985). Las luchas de clases en Francia de 1848 a 1850 y El Dieciocho Brumario de Luis Bonaparte. Madrid, Espasa-Calpe, 349p.

Mayo, Elton (1945). The social problems of an Industrial Civilization. Boston, Harvard Uni versity, 150p.

McGregor, Douglas. (1957). Proceedings of the Fifth Anniversary Convocation of the School of Industrial Management, The Human Side of Enterprise. Massachusetts Institute of Technology, 125p.

Meroño, A. (2005). Tecnologías de información y gestión del conocimiento. Integración de un sistema. En: Economía Industrial, Vol. 357, pp. 107-116.

Miller, Eric J. y Rice, A. K. (1967) Systems of Organization. London, Tavistock Publications. 289 p.

Mintzberg, Henry (2001). Diseño de organizaciones eficientes. Argentina: El Ateneo, 365p.

Mintzberg, Henry y Heyden, Ludo Van der (2006). Revisando el concepto de organización. En: Harvard Deusto Business Review, Vol. 150, pp. 4-13.

Mintzberg, Henry y McHugh, Alexandra (1985). Strategy Formation in Adhocracy. En: Administrative Science Quarterly, Vol. 30, N. ${ }^{\circ}$ 2, June, pp. 160-197.

Morgan, Gareth (1998). Imágenes de la Organización. México, Alfaomega, 450p.

Murillo, Guillermo; Zapata, Álvaro; Martínez, Jenny; Ávila, Hernán; Salas, Jairo; González, Carlos Hernán y López, Hernán. (2008b). Teorías contemporáneas de la organización y el management. Bogotá, ECOE Ediciones y Universidad del Valle, 280p.

Murillo, Guillermo; Zapata, Álvaro; Martínez, Jenny; Ávila, Hernán; Salas, Jairo; y López, Hernán (2008a). Teorías clásicas de la organización y el management. Bogotá, ECOE Ediciones y Universidad del Valle, 2a edición, 280p.

Semestre Económico, volumen 15, №. 32, pp. 161-184 • ISSN 0120-6346, julio-diciembre de 2012, Medellín, Colombia 
Nieto, Mauricio (2007). Los clúster del conocimiento. Una importante herramienta para la transformación productiva. En: Revista Escuela de Administración de Negocios, N. ${ }^{\circ}$ 61, septiembre-diciembre, pp. 5-25.

Nonaka, Ikujiro y Takeuchi, Hitoraka (1999). La organización creadora de conocimiento. México, Oxford, 220p.

Nonaka, Ikujiro y Takeuchi, Hitoraka. (1995). The knowledge creating company. How Japanese companies create the dynamics of innovation. New York, Oxford University Press, 280p.

Nonaka, Ikujiro y Toyama, R. (2003). The knowledge-creating theory revisited Knowledge creation as a synthesizing process. En: Knowledge Management Research y Practice, Vol. 1, N. ${ }^{\circ} 1$, pp. 2-10.

Ouchi, William (1981). Theory, How American Business can meet the Japanese challenge, Reading, Massachusetts, Addison-Wesley, 290p.

Parsons, Talcott (1968). La estructura de la acción social. Madrid: Guadarama, 371p.

Patrus, Robert (2004). La empresa ética, ¿̇un nuevo paradigma? Condiciones, desafíos y riesgos del desarrollo de la Business Ethics. Tesis doctoral. Madrid, Departamento de Filosofía Moral y Política, Universidad Complutense de Madrid. 450 p.

Perrow, Charles (1972). Análisis organizacional: una visión sociológica. México: CECSA, 267 p.

Peters, Tom y Waterman, R. (1982). In Search of excellence, New York, Harper and Row, 240p.

Porter, Michael (1980). Competitive Strategy, Free Press, New York, 450p.

Porter, Michael (1985). Competitive Advantage, Free Press, New York, 420p.

Porter, Michael (1998). The Competitive Advantage of Nations. Free Press, New York, 390p.

Prahalad, Coimbatore K. (1994). Strategy as a field, Why Search for a New Paradigm. En: Strategic Management Journal, Vol. 15, pp. 5-16.

Prahalad, Coimbatore K. (2004). The Fortune at the Bottom of the Pyramid, Eradicating Poverty through Profits. Wharton School Publishing, 230p.

Prahalad, Coimbatore K. (2006). The location barrier [around innovation] is finally broken. Keynote address to the Software 2006 Conference, IT Conversations. A conversations network channel. Dr. Prahalad. University of Michigan. Emerging Hi-Tech Ecosystems, Software Conference. [En líneal Recuperado en octubre de 2010, disponible en http.// itc.conversationsnetwork.org/shows/detail1030.html.

Prahalad, Coimbatore K. y Hamel, Gary (1990). The core competence of the corporation. En: Harvard Business Review, Vol. 90, N.³, pp. 79-91, Mayo-Junio.

Prahalad, Coimbatore K. y Gibson, R. (1997). Rethinking the future. Rethinking the Future, Rethinking Business, Principles, Competition, Control y Complexity, Leadership, Markets, and the World. Rowan Gibson (Ed.). London, Nicholas Brealey Purshing, 430p. 
La organización y su adaptación a las tecnologías de la información y la comunicación en procesos de gestión del conocimiento

Prahalad, Coimbatore K. y Hamel, Gary (1994). Competing for the future. Boston, 320p.

Prahalad, Coimbatore K. y Hamel, Gary (1998). La estrategia como objeto de estudio, ¿por qué buscar un nuevo paradigma? En: Enciclopedia Práctica de Management, Planeta D'Agostini, pp. 30-37.

Prahalad, Coimbatore K. y Krishnan, M.S. (2007). The New Age of innovation. Boston, Massachusetts, Harvard Business School Press, 290p.

Prahalad, Coimbatore K. y Ramaswamy, Venkat. (2004a). Co-creation experiences: The next practice in value creation. Journal of Interactive Marketing, Vol. 18, N. ${ }^{\circ} 3$, Verano, pp. $5-14$.

Prahalad, Coimbatore K., y Ramaswamy, Venkat. (2004b). The Future of Competition: Co-Creating Unique Value with Customers. Boston: Harvard Business School Press.

Prahalad, Coimbatore K., y Ramaswamy, Venkat. (2005). Building New Strategic Capital for Co-Creation. Strategy + Business, forthcoming, 220 p.

Rueda, Gladys Elena (2012). Influencia de la cultura organizacional, la gestión del conocimiento y el capital tecnológico en la producción científica. Aplicación a grupos de investigación adscritos a Universidades en Colombia. Tesis Doctoral. Programa Doctoral Integración de las Tecnologías de la Información en las Organizaciones, Valencia, Universitat Politecnica de Valencia, 540p.

Selznick, Philip (1957). Leadership in administration. A sociological interpretation. Berkeley, London, University of California Press, 290p.

Simmel, Georg (1977). Sociología, Estudios sobre las formas de socialización. En: Revista de Occidente, Madrid, pp. 17-34.

Simon, Herbert (1977). The new science of management decision. Englewood Cliffs, New Jersey, Prentice-Hall, $3^{a}$ edición, 390p.

Skyrme, D. (2002). Knowledge Management: Approaches and Policies. [En línea] Disponible en http://www.kmadvantage.com/docs/km_articlesKM___Approaches_and_Policies. pdf. [enero de 2010].

Smith, Adam (1983). La riqueza de las naciones: Investigación de la naturaleza y causas de la riqueza de las naciones. México, Fondo de Cultura Económica, 3 Volúmenes, 917 p.

Spencer, Herbert (1882). The study of sociology. New York, D. Appleton, 340p.

Sveiby, Karl-Erik (1997). The New Organizacional Wealth, Managing and Measuring Knowledge-Based Assets. San Francisco, Berrett-Koehler, 290p.

Tapscott, Don (2006). Wikinomics, How Mass Collaboration Changes Everyting, Portafolio Handcover. 325p.

Semestre Económico, volumen 15, №. 32, pp. 161-184 • ISSN 0120-6346, julio-diciembre de 2012, Medellín, Colombia 
Jenny Martínez Crespo • Lillyana María Giraldo Marín

Taylor, Frederick Winslow (1961). Administración científica. México, Herrera Hermanos, 140p.

Tissen, R., Andriessen, D., y Lekanne, F. (2000). El valor del conocimiento para aumentar el rendimiento en las empresas. Madrid: Prentice Hall.

Weber, Max (1964). Economía y Sociedad. México, FCE, 480p.

Woodward, Joan (1965). Industrial Organization. Theory y Practice. Oxford, Oxford University Press, 180p.

Zapata, Álvaro (2008). Análisis y diseño organizacional. De la estructura funcional a la organización vacía. Cali, Universidad del Valle, 320p.

Zapata, Álvaro, Murillo, Guillermo y Martínez, Jenny (2006). Organización y Management. Naturaleza, objeto, método, investigación y enseñanza. Cali, Editorial Universidad del Valle, 295p. 